\title{
ON THE COHOMOLOGY OF SOME HOPF ALGEBROIDS AND HATTORI-STONG THEOREMS
}

\author{
ANDREW BAKER \\ (communicated by Hvedri Inassaridze)
}

\begin{abstract}
We apply group cohomological methods to calculate the cohomology of $K(n)_{*} B P$ as a $K(n)_{*} K(n)$-comodule, recovering recent results of Hovey and Sadofsky. As applications we determine the Chromatic Spectral Sequence for BP based on Johnson and Wilson's $E(n)$, showing the relationship to some generalizations of the classical HattoriStong Theorem and determine the change of Hopf algebroid spectral sequence associated with the natural map $B P \longrightarrow E(n)$, extending calculations of Clarke for the Todd orientation $M U \longrightarrow K U$.
\end{abstract}

\section{Introduction}

In an interesting paper [4], Hovey and Sadofsky showed amongst other things that the Chromatic Spectral Sequence associated to a $p$-local $v_{n}$-periodic ring spectrum such as Johnson and Wilson's $E(n)$ is obtained by taking the 0 to $n$th columns of the Chromatic Spectral Sequence associated with $B P$-theory. They also identified $K(n)_{*} B P$ as a comodule over the Hopf algebroid $K(n)_{*} K(n)=K(n)_{*}(E(n))$. In [2], we also analysed the structure of algebras of the form

$$
R_{*} K(n)=R_{*} \underset{B P_{*}}{\otimes} B P_{*} K(n),
$$

where $R_{*}$ is a $B P_{*}$-algebra such as $B P_{*}$ or $E(n)_{*}$, and used our results to give an alternative approach to such results.

Clarke [3] discussed the change of Hopf algebroid spectral sequence associated to the Todd orientation $\tau: M U \longrightarrow K U$ and showed that

$$
\operatorname{Ext}_{K U_{*} K U}^{1, *}\left(K U_{*}, K U_{*} M U\right) \neq 0,
$$

despite the Hattori-Stong Theorem, which was taken as evidence that the $K U$-theory Adams Spectral Sequence might be trivial since

$$
M U_{*} \cong \operatorname{Ext}_{K U_{*} K U}^{0, *}\left(K U_{*}, K U_{*} M U\right) .
$$

Of course, in the two decades since the publication of [3], our understanding of the target $\pi_{*}\left(\mathrm{~L}_{K U} M U\right)$ of this spectral sequence has improved and such a result no longer appears surprising.

In this paper we use methods of Galois cohomology to rederive certain cohomology groups over the Hopf algebra $K(n)_{*} K(n)$ and explore some consequences of our current knowledge of the Chromatic Spectral Sequence for

$$
\operatorname{Ext}_{E(n)_{*} E(n)}^{*, *}\left(E(n)_{*}, E(n)_{*} B P\right),
$$

Received 5 January 2000, revised 15 February 2000; published on 9 March 2000.

1991 Mathematics Subject Classification: Primary 55N20 55N22 55T15

Key words and phrases: Hopf algebroid, comodule, Brown-Peterson spectrum, Johnson-Wilson spectrum, Galois cohomology

(C) 2000, Andrew Baker. Permission to copy for private use granted. 
and compute this completely, relating the result to a change of Hopf algebroid spectral sequence

$$
\begin{aligned}
& \mathrm{E}_{2}^{s, t, *}=\operatorname{Ext}_{B P_{*} B P}^{r, *}\left(B P_{*}, \operatorname{Ext}_{E(n)_{*} E(n)}^{s, *}\left(E(n)_{*}, E(n)_{*} B P\right)\right) \\
& \Longrightarrow \operatorname{Ext}_{E(n)_{*} E(n)}^{s+t, *}\left(E(n)_{*}, E(n)_{*}\right) .
\end{aligned}
$$

This generalizes Clarke's work and we show that there are always non-zero elements in

$$
\operatorname{Ext}_{E(n)_{*} E(n)}^{n, *}\left(E(n)_{*}, E(n)_{*}\right)
$$

playing an analogous rôle to the elements he identified when $n=1$. We also state some generalizations of the Hattori-Stong Theorem following from our analysis.

I would like to thank Ken Brown, Francis Clarke, Steve Cohen, Mark Hovey and Hal Sadofsky for their help and inspiration, and also the referee of an earlier version whose perceptive comments were invaluable.

\section{Review of some results of Hovey and Sadofsky}

Throughout this paper, we follow well established (but nevertheless unfortunate) fashion in writing

$$
K(n)_{*} K(n)=K(n)_{*}(E(n)) \varsubsetneqq K(n)_{*}(K(n)) .
$$

By $\left[4\right.$, theorem B], we have a good description of the $K(n)_{*} K(n)$-comodule structure of $K(n)_{*} B P$; note that this is not claimed to give a description of the $K(n)_{*} K(n)$-comodule algebra structure.

Theorem 1.1. There is an isomorphism of left $K(n)_{*} K(n)$-comodules

$$
K(n)_{*} B P \cong K(n)_{*} E(n)\left[w_{i}: i \geqslant 1\right],
$$

where $w_{i} \in K(n)_{2 p^{n}\left(p^{i}-1\right)} B P$ is primitive with respect to the coaction.

$K(n)_{*} B P$ is an extended $K(n)_{*} K(n)$-comodule, so for $s>0$,

$$
\operatorname{Ext}_{K(n)_{*} K(n)}^{s, *}\left(K(n)_{*}, K(n)_{*} B P\right)=0 .
$$

It is straightforward to deduce the following consequence.

Corollary 1.2. $E(n)_{*} B P /\left(p^{\infty}, v_{1}^{\infty}, \ldots, v_{n-1}^{\infty}\right)$ is an extended left $E(n)_{*} E(n)$-comodule,

$$
\begin{aligned}
E(n)_{*} B P /\left(p^{\infty}, v_{1}^{\infty}, \ldots,\right. & \left.v_{n-1}^{\infty}\right) \\
& \cong E(n)_{*} E(n) \underset{E(n)_{*}}{\otimes} E(n)_{*} /\left(p^{\infty}, v_{1}^{\infty}, \ldots, v_{n-1}^{\infty}\right)\left[\widetilde{w}_{i}: i \geqslant 1\right],
\end{aligned}
$$

where $\widetilde{w}_{i}$ has degree $2 p^{n}\left(p^{i}-1\right)$ and is coaction primitive. Hence for $s>0$,

$$
\operatorname{Ext}_{E(n)_{*} E(n)}^{s, *}\left(E(n)_{*}, E(n)_{*} B P /\left(p^{\infty}, v_{1}^{\infty}, \ldots, v_{n-1}^{\infty}\right)\right)=0 .
$$

In $[4$, theorem 5.1] the Chromatic Spectral Sequence $\operatorname{CSS}(S ; E(n))$ based on $E(n)$ for the sphere spectrum $S$ was shown to be obtained by truncating that based on $B P$. There is an obvious generalization to the Chromatic Spectral Sequence $\operatorname{CSS}(X ; E(n))$ of a connective torsion free spectrum $X$ based on $E(n)$ and converging to

$$
\operatorname{Ext}_{E(n)_{*} E(n)}^{*, *}\left(E(n)_{*}, E(n)_{*} X\right)
$$

Recall that the $\mathrm{E}_{1}$-term of the Chromatic Spectral Sequence $\operatorname{CSS}(X ; B P)$ based on $B P$ and converging to

$$
\operatorname{Ext}_{B P_{*} B P}^{*, *}\left(B P_{*}, B P_{*} X\right)
$$


has the form

$$
\mathrm{E}_{1}^{n, t, *}(X ; B P)=\operatorname{Ext}_{E(n)_{*} E(n)}^{t, *}\left(E(n)_{*}, E(n)_{*} X /\left(p^{\infty}, v_{1}^{\infty}, \ldots, v_{n-1}^{\infty}\right)\right) .
$$

Theorem 1.3. In the $\mathrm{E}_{1}$-term of the spectral sequence $\operatorname{CSS}(X ; E(n))$,

$$
\mathrm{E}_{1}^{k, t, *}(X ; E(n))= \begin{cases}\mathrm{E}_{1}^{k, t, *}(X ; B P) & \text { if } k \leqslant n, \\ 0 & \text { if } k>n,\end{cases}
$$

and the differential $\mathrm{d}_{1}^{k, t, *}$ with domain $\mathrm{E}_{1}^{k, t, *}(X ; E(n))$ is obtained from the corresponding differential of $\operatorname{CSS}(X ; B P)$ whenever $\mathrm{E}_{1}^{k, t, *}(X ; E(n)) \neq 0$.

When $X=B P$, Corollary 1.2 implies that the only possible non-zero differential in each of the spectral sequences $\operatorname{CSS}(B P ; E(n))$ and $\operatorname{CSS}(B P ; B P)$ is $\mathrm{d}_{1}$.

\section{The cohomology of $K(n)_{*} B P$ as Galois cohomology}

Our aim in this section is to reprove the results of Theorem 1.1 and Corollary 1.2 using our previous work $[\mathbf{1}, \mathbf{2}]$ and making use of continuous cohomology of profinite groups.

From now on, let $R_{*}$ be an algebra over $\mathbb{F}_{p^{n}} \otimes B P_{*}$ annihilated by $I_{n}$ and in which there is a unit $u$ satisfying $v_{n}=u^{p^{n}-1}$. In our applications to $K(n)_{*} B P$ we will take

$$
R_{*}=\mathbb{F}_{p^{n}} \otimes\left(v_{n}^{-1} B P_{*} / I_{n}\right)[u] /\left(u^{p^{n}-1}-v_{n}\right),
$$

but for completeness we give the general version.

We may form the $R_{*}$-algebra

$$
R_{*} K(n)=R_{*} \underset{B P_{*}}{\otimes} B P_{*} B P \underset{B P_{*}}{\otimes} K(n)_{*} .
$$

From [2, Theorem A.3] we have

Theorem 2.1. $R_{*} K(n)$ is a free $R_{*}$-module. Moreover, there is an exhaustive filtration by subalgebras

$$
R_{*}=R_{*} K(n)^{(1)} \subseteq R_{*} K(n)^{(2)} \subseteq \cdots \subseteq R_{*} K(n)^{(k)} \subseteq \cdots \subseteq R_{*} K(n)
$$

in which each extension $R_{*} K(n)^{(k)} \longrightarrow R_{*} K(n)^{(k+1)}$ is a free $R_{*} K(n)^{(k)}$-module and a Galois extension of Artin-Schreier type with Galois group $\mathbb{F}_{p^{n}}$.

There is an action of the Morava stabiliser group $\mathbb{S}_{n}$ on $R_{*} K(n)$ with respect to which

$$
R_{*} K(n)^{(k)}=R_{*} K(n)_{*}^{\mathbb{S}_{*}^{[k]}}
$$

the fixed point set of the closed subgroup

$$
\mathbb{S}_{n}^{[k]}=\left\{1+\sum_{k \leqslant r} \alpha_{r} S^{r}: \forall r, \alpha_{r}^{p^{n}}=\alpha_{r}\right\} \leqslant \mathbb{S}_{n} .
$$

Here an extension $A \longrightarrow B$ of commutative unital $\mathbb{F}_{q}$-algebras is of Artin-Schreier type if

$$
B=A[t] /\left(t^{q}-t-a\right)
$$

for some $a \in A$; its Galois group is $\operatorname{Gal}(B / A)=\mathbb{F}_{q} \leqslant \operatorname{Aut}_{A}(B)$ where $\gamma \in \mathbb{F}_{q}$ acts by $\gamma \cdot f(t)=f(t+\gamma)$. Notice that $B$ is a free $A$-module of $\operatorname{rank} q$ and $B^{\operatorname{Gal}(B / A)}=A$, however in general $\operatorname{Gal}(B / A) \neq \operatorname{Aut}_{A}(B)$ (consider for example the case where $a=0$ ).

We will apply this result to calculate the continuous cohomology of $B P_{*} K(n)$ with respect to with action of $\mathbb{S}_{n}$, where this continuous cohomology is equivalent to the cohomology over the $K(n)_{*}$-Hopf algebra $K(n)_{*} K(n)$. Further information the relationship of the action of $\mathbb{S}_{n}$ and the $K(n)_{*} K(n)$-comodule structure can be found in $[\mathbf{1}]$. 
Theorem 2.2. The continuous cohomology of $R_{*} K(n)$ with respect to $\mathbb{S}_{n}$ is given by

$$
\mathrm{H}_{c}^{s}\left(\mathbb{S}_{n} ; R_{*} K(n)\right)= \begin{cases}R_{*} & \text { if } s=0, \\ 0 & \text { if } s \neq 0 .\end{cases}
$$

In order to apply Galois cohomological machinery we will require two technical Galois theoretic results surely known to experts but for which we know of no convenient references.

Lemma 2.3 (Normal Basis Theorem for Artin-Schrier extensions). Let $A$ be a commutative unital $\mathbb{F}_{q}$-algebra. Let $B=A[t] /(f(t))$ be the Artin-Schrier extension associated with the polynomial $f(X)=X^{q}-X+a \in A[X]$. Then the elements $(t+\gamma)^{q-1}\left(\gamma \in \mathbb{F}_{q}\right)$ form an $A$-basis for $B$. Hence $B$ is a free $A[\operatorname{Gal}(B / A)]$-module.

Proof. We will show that in the $\mathbb{F}_{q}$-vector subspace spanned by the monomials $t^{k}(0 \leqslant k \leqslant$ $q-1)$, the stated elements form a basis. Let $\omega \in \mathbb{F}_{q}^{\times}$be a generator, so the non-zero elements of $\mathbb{F}_{q}$ are expressible as powers of $\omega$.

By the Binomial Theorem,

$$
(t+\gamma)^{q-1}=\sum_{0 \leqslant r \leqslant q-1}\left(\begin{array}{c}
q-1 \\
r
\end{array}\right) \gamma^{r} t^{q-1-r},
$$

so it suffices to show the non-singularity of the matrix

$$
\left[\begin{array}{ccccc}
1 & 0 & 0 & \cdots & 0 \\
1 & \left(\begin{array}{c}
q-1 \\
1
\end{array}\right) & \left(\begin{array}{c}
q-1 \\
2
\end{array}\right) & \cdots & 1 \\
1 & (q-1) \omega & \left(\begin{array}{c}
q-1 \\
1
\end{array}\right) \omega^{2} & \cdots & 1 \\
1 & \left(\begin{array}{c}
q-1 \\
1
\end{array}\right) \omega^{2} & \left(\begin{array}{c}
q-1 \\
2
\end{array}\right) \omega^{4} & \cdots & 1 \\
1 & \left(\begin{array}{c}
q-1 \\
1
\end{array}\right) \omega^{3} & \left(\begin{array}{c}
q-1 \\
2
\end{array}\right) \omega^{6} & \cdots & 1 \\
\vdots & \vdots & \vdots & \ddots & \vdots \\
1 & \left(\begin{array}{c}
q-1 \\
1
\end{array}\right) \omega^{q-2} & \left(\begin{array}{c}
q-1 \\
2
\end{array}\right) \omega^{2(q-2)} & \cdots & 1
\end{array}\right]
$$

whose determinant is

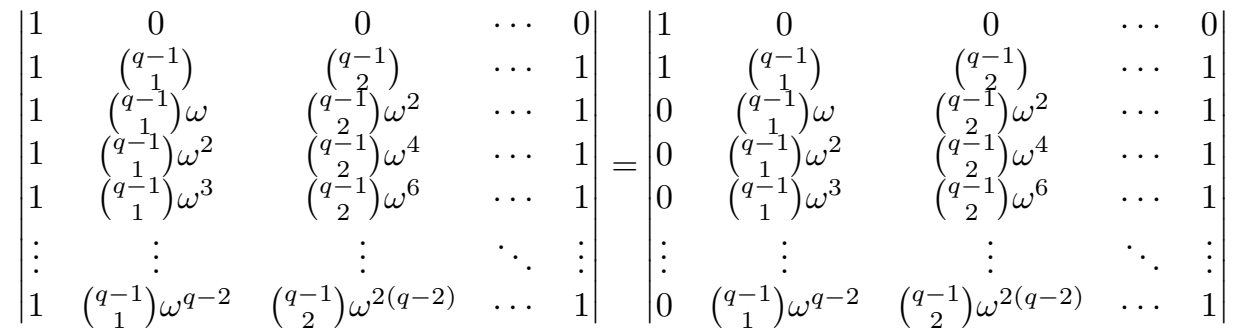

$$
\begin{aligned}
& =\left(\begin{array}{c}
q-1 \\
1
\end{array}\right)\left(\begin{array}{c}
q-1 \\
2
\end{array}\right) \cdots\left(\begin{array}{c}
q-1 \\
q-2
\end{array}\right)\left|\begin{array}{ccccc}
1 & 0 & 0 & \cdots & 0 \\
0 & 1 & 1 & \cdots & 1 \\
0 & \omega & \omega^{2} & \cdots & 1 \\
0 & \omega^{2} & \omega^{4} & \cdots & 1 \\
0 & \omega^{3} & \omega^{6} & \cdots & 1 \\
\vdots & \vdots & \vdots & \ddots & \vdots \\
0 & \omega^{q-2} & \omega^{2(q-2)} & \cdots & 1
\end{array}\right| \\
& =\left(\begin{array}{c}
q-1 \\
1
\end{array}\right)\left(\begin{array}{c}
q-1 \\
2
\end{array}\right) \cdots\left(\begin{array}{c}
q-1 \\
q-2
\end{array}\right) \prod_{0 \leqslant r<s \leqslant q-2}\left(\omega^{r}-\omega^{s}\right) .
\end{aligned}
$$

This is non-zero because the binomial coefficients satisfy

$$
\left(\begin{array}{c}
q-1 \\
r
\end{array}\right) \equiv\left(\begin{array}{c}
p-1 \\
r_{0}
\end{array}\right)\left(\begin{array}{c}
p-1 \\
r_{1}
\end{array}\right) \cdots\left(\begin{array}{c}
p-1 \\
r_{d}
\end{array}\right) \bmod (p)
$$


where $r=r_{0}+r_{1} p+\cdots+r_{m} p^{m}$ is the $p$-adic expansion of $r$, and the factors are never divisible by $p$. So the $(t+\gamma)^{q-1}$ form a basis in place of the $t^{k}$. As this is true over $\mathbb{F}_{q}$, it is also true over $A$.

The next result will allow an inductive calculation of cohomology in the situation of interest.

Lemma 2.4. Let $A \subseteq B \subseteq C$ be a tower of commutative unital rings. Let $G \leqslant \operatorname{Aut}_{A}(C)$ be finite and $N \triangleleft G$ with $C^{G}=A, C^{N}=B$, and $C \cong B[N]$ as a $B[N]$-module. Then

$$
\mathrm{H}^{*}(G ; C) \cong H^{*}(G / N ; B) \text {. }
$$

Proof. There is a Hochschild-Serre Spectral Sequence [7, chapter II §4],

$$
\mathrm{E}_{2}^{r, s}=\mathrm{H}^{r}\left(G / N ; \mathrm{H}^{s}(N ; C)\right) \Longrightarrow \mathrm{H}^{r+s}(G ; C) .
$$

Since

$$
\mathrm{H}^{*}(N ; C) \cong \mathrm{H}^{*}(N ; B[N])=\mathrm{H}^{0}(N ; B[N]) \cong \mathrm{H}^{0}(N ; C)=B
$$

the $\mathrm{E}_{2}$-term is trivial except for $\mathrm{E}_{2}^{r, 0}=\mathrm{H}^{r}(G / N ; B)$, so the result follows.

Proof of Theorem 2.2. From the proof of [2, theorem A.3], for $k \geqslant 1, R_{*} K(n)^{(k)}$ is a free $R_{*}$-module with a basis consisting of the monomials

$$
t_{1}^{s_{1}} t_{2}^{s_{2}} \cdots t_{k-1}^{s_{k-1}}, \quad\left(0 \leqslant s_{k} \leqslant p^{n}-1\right) .
$$

For each of these basis elements, the stabilizer is an open subgroup of $\mathbb{S}_{n}$ since it contains an open subgroup of the form $\mathbb{S}_{n}^{[k]}$. Hence the action of $\mathbb{S}_{n}$ on $R_{*} K(n)$ is proper in the sense that the stabilizer of each point is an open subgroup and this allows us to apply the cohomological machinery of Shatz [7] to $R_{*} K(n)$. In particular, [7, chapter 3 theorem 7] gives

$$
\mathrm{H}_{c}^{*}\left(\mathbb{S}_{n} ; R_{*} K(n)\right)=\underset{k}{\lim } \mathrm{H}^{*}\left(\mathbb{S}_{n} / \mathbb{S}_{n}^{[k]} ; R_{*} K(n)^{(k)}\right) .
$$

To complete the proof we will show by induction on $k \geqslant 1$ that

$$
\mathrm{H}^{*}\left(\mathbb{S}_{n} / \mathbb{S}_{n}^{[k]} ; R_{*} K(n)^{(k)}\right)=\mathrm{H}^{0}\left(\mathbb{S}_{n} / \mathbb{S}_{n}^{[k]} ; R_{*} K(n)^{(k)}\right)=R_{*} .
$$

For $k=1$, the result is certainly true. Suppose that it holds for some $k=m \geqslant 1$. The extension $R_{*} K(n)^{(m)} \subseteq R_{*} K(n)^{(m+1)}$ satisfies the conditions of Lemma 2.3 and so we can also apply Lemma 2.4 with

$$
A=R_{*}, B=R_{*} K(n)^{(m)}, C=R_{*} K(n)^{(m+1)}, G=\mathbb{S}_{n} / \mathbb{S}_{n}^{[m+1]}, N=\mathbb{S}_{n}^{[m]} / \mathbb{S}_{n}^{[m+1]} .
$$

This yields

$$
\mathrm{H}^{*}\left(\mathbb{S}_{n} / \mathbb{S}_{n}^{[m+1]} ; R_{*} K(n)^{(m+1)}\right)=\mathrm{H}^{*}\left(\mathbb{S}_{n} / \mathbb{S}_{n}^{[m]} ; R_{*} K(n)^{(m)}\right)=R_{*},
$$

which verifies the inductive step.

Remark. Since $\mathbb{S}_{n} / \mathbb{S}_{n}^{[k]}$ is a $p$-group, the vanishing of $\mathrm{H}^{s}\left(\mathbb{S}_{n} / \mathbb{S}_{n}^{[k]} ; R_{*} K(n)^{(k)}\right)$ for $s>0$ actually implies that $R_{*} K(n)^{(k)}$ is a projective $\mathbb{F}_{q}\left[\mathbb{S}_{n} / \mathbb{S}_{n}^{[k]}\right]$-module and hence is free because this group algebra is local. Of course, if we knew an explicit permutation basis we could avoid the cohomological calculations.

\section{Corollary 2.5.}

$$
\operatorname{Ext}_{K(n)_{*} K(n)}^{s, *}\left(K(n)_{*}, K(n)_{*} B P\right)= \begin{cases}v_{n}^{-1} B P_{*} / I_{n} & \text { if } s=0 \\ 0 & \text { if } s \neq 0\end{cases}
$$


Proof. We apply the Theorem in the case

$$
R_{*}=\mathbb{F}_{p^{n}} \otimes\left(v_{n}^{-1} B P_{*} / I_{n}\right)[u] /\left(u^{p^{n}-1}-v_{n}\right) .
$$

A complication comes from the fact that we have extended $v_{n}^{-1} B P_{*} / I_{n}$ to $R_{*}$ by tensoring with $\mathbb{F}_{p^{n}}$ then adjoining a $\left(p^{n}-1\right)$ st root of $v_{n}$. This is a Galois extension whose Galois group $\Gamma_{n}$ is identified with $\mathbb{F}_{p^{n}}^{\times} \rtimes \mathbb{Z} / n$. Furthermore,

$$
\operatorname{Ext}_{K(n)_{*} K(n)}^{s, *}\left(K(n)_{*}, K(n)_{*} B P\right)=\mathrm{H}_{c}^{*}\left(\mathbb{S}_{n} ; R_{*} K(n)\right)^{\Gamma_{n}} .
$$

All of this is straightforward given the well understood identification of $\mathbb{S}_{n}$ with the group of strict units in the maximal order $\mathcal{O}_{n}$ of a certain $p$-adic division algebra $\mathbb{D}_{n}$. In fact there is a proper action on $R_{*} K(n)$ of a semi-direct product extension of $\mathbb{S}_{n}, \mathcal{O}_{n}^{\times} \rtimes \mathbb{Z} / n$, where the full group of units $\mathcal{O}_{n}^{\times}$can itself be viewed as an extension

$$
1 \rightarrow \mathbb{S}_{n} \longrightarrow \mathcal{O}_{n}^{\times} \longrightarrow \mathbb{F}_{p^{n}}^{\times} \rightarrow 1 .
$$

We will use the notation $u_{k}=\eta_{\mathrm{L}}\left(v_{k}\right), v_{k}=\eta_{\mathrm{R}}\left(v_{k}\right)$, where

$$
E(n)_{*} \stackrel{\eta_{\mathrm{L}}}{\longrightarrow} E(n)_{*} B P \stackrel{\eta_{\mathrm{R}}}{\stackrel{v^{2}}{\longrightarrow}} B P_{*}
$$

are the left and right units. As $v_{k}$ is a coaction primitive, multiplication by $v_{k}$ is a homomorphism of $E(n)_{*} E(n)$-comodules $E(n)_{*} B P \longrightarrow E(n)_{*} B P$. Hence $E(n)_{*} B P /\left(p^{r_{0}}, v_{1}^{r_{1}}, \cdots, v_{n-1}^{r_{n-1}}\right)$ is a quotient $E(n)_{*} E(n)$-comodule of $E(n)_{*} B P$. As $I_{n}=\left(p, v_{1}, \ldots, v_{n-1}\right) \triangleleft B P_{*}$ is an invariant ideal,

$$
K(n)_{*} B P=E(n)_{*} B P /\left(p, v_{1}, \ldots, v_{n-1}\right)=E(n)_{*} B P /\left(p, u_{1}, \ldots, u_{n-1}\right) .
$$

Theorem 2.6. Suppose that $1 \leqslant r_{k} \leqslant \infty$ for $k=0,1, \ldots, n-1$. Then

$$
\begin{aligned}
\operatorname{Ext}_{E(n)_{*} E(n)}^{s, *}\left(E(n)_{*}, E(n)_{*} B P /\left(p^{r_{0}}, v_{1}^{r_{1}}, \ldots,\right.\right. & \left.\left.v_{n-1}^{r_{n-1}}\right)\right) \\
& = \begin{cases}v_{n}^{-1} B P_{*} /\left(p^{r_{0}}, v_{1}^{r_{1}}, \ldots, v_{n-1}^{r_{n-1}}\right) & \text { if } s=0, \\
0 & \text { if } s \neq 0 .\end{cases}
\end{aligned}
$$

Proof. Suppose that all of the exponents $r_{k}$ are finite. We will prove the result by induction on the number

$$
\operatorname{excess}\left(r_{0}, r_{1}, \ldots, r_{n-1}\right)=\sum_{k=0}^{n-1}\left(r_{k}-1\right) \geqslant 0 .
$$
0 .

Corollary 2.5 implies that the result is true for the unique sequence $(1,1, \ldots, 1)$ of excess

Now suppose that it holds whenever excess $\left(r_{0}, r_{1}, \ldots, r_{n-1}\right)<m$. Then for at least one value of $\ell$ in the range $0 \leqslant \ell \leqslant(n-1)$ we have $r_{\ell}>1$. Let $\left(r_{0}^{\prime}, r_{1}^{\prime}, \ldots, r_{n-1}^{\prime}\right)$ be the exponent sequence for which

$$
r_{k}^{\prime}= \begin{cases}r_{\ell}-1 & \text { if } k=\ell, \\ r_{k} & \text { if } k \neq \ell .\end{cases}
$$

Then there is an exact sequence of $E(n)_{*} E(n)$-comodules

$$
\begin{aligned}
0 \rightarrow E(n)_{*} B P /\left(p^{r_{0}}, v_{1}^{r_{1}}, \ldots, v_{\ell-1}^{r_{\ell-1}}, v_{\ell}, v_{\ell+1}^{r_{\ell+1}}, \ldots, v_{n-1}^{r_{n-1}}\right) & \longrightarrow \\
E(n)_{*} B P /\left(p^{r_{0}}, v_{1}^{r_{1}}, \ldots, v_{n-1}^{r_{n-1}}\right) & \longrightarrow E(n)_{*} B P /\left(p^{r_{0}^{\prime}}, v_{1}^{r_{1}^{\prime}}, \ldots, v_{n-1}^{r_{n-1}^{\prime}}\right) \rightarrow 0 .
\end{aligned}
$$

Here the left-hand monomorphism is induced from the homomorphism of comodules

$$
E(n)_{*} B P \longrightarrow E(n)_{*} B P /\left(p^{r_{0}}, v_{1}^{r_{1}}, \ldots, v_{n-1}^{r_{n-1}}\right) ; \quad x \longmapsto v_{\ell}^{r_{\ell}-1} x .
$$


The exponent sequences of the left and right hand terms each have excess less that $m$ and so we obtain a long exact sequence in Ext yielding the result for

$$
E(n)_{*} B P /\left(p^{r_{0}}, v_{1}^{r_{1}}, \ldots, v_{n-1}^{r_{n-1}}\right) .
$$

Hence the result for sequences of arbitrary excess follows by induction.

When some of the $r_{k}$ are infinite the result is proved by using appropriate colimits over finite exponent cases under obvious inclusions maps.

\section{Some generalizations of the Hattori-Stong Theorem}

Recall the well-known fact that

$$
\operatorname{Ext}_{B P_{*} B P}^{s, *}\left(B P_{*}, B P_{*} B P\right)= \begin{cases}B P_{*} & \text { if } s=0 \\ 0 & \text { if } s \neq 0 .\end{cases}
$$

Using this we can deduce that $\operatorname{CSS}(B P ; B P)$ collapses at $\mathrm{E}_{2}=\mathrm{E}_{\infty}$. This gives

Theorem 3.1 (Hattori-Stong Theorem in $E(n)$-theory). For each $n \geqslant 1$, the $E(n)$ Hurewicz homomorphism $B P_{*} \longrightarrow E(n)_{*} B P$ is a split monomorphism.

The following observation will prove useful in the proof.

Lemma 3.2. For $n \geqslant 1$,

$$
\operatorname{Ext}_{E(n)_{*} E(n)}^{0, *}\left(E(n)_{*}, E(n)_{*} B P\right) \subseteq E(n)_{*} B P
$$

is the kernel of the $\mathbb{Z}_{(p)}$-homomorphism

$$
\psi-1 \otimes \mathrm{Id}: E(n)_{*} B P \longrightarrow E(n)_{*} E(n) \underset{E(n)_{*}}{\otimes} E(n)_{*} B P
$$

in which the domain and codomain are torsion-free; hence it is an additive summand.

Proof of Theorem 3.1. There is an exact sequence

$$
\begin{aligned}
0 \rightarrow \operatorname{Ext}_{B P_{*} B P}^{0, *}\left(B P_{*}, B P_{*} B P\right) & \longrightarrow \operatorname{Ext}_{E(0)_{*} E(0)}^{0, *}\left(E(0)_{*}, E(0)_{*} B P\right) \\
& \stackrel{\mathrm{d}_{1}}{\longrightarrow} \operatorname{Ext}_{E(1)_{*} E(1)}^{0, *}\left(E(1)_{*}, E(1)_{*} B P /\left(p^{\infty}\right)\right) \stackrel{\mathrm{d}_{1}}{\longrightarrow} \cdots
\end{aligned}
$$

By the well known fact that

$$
\operatorname{Ext}_{E(0)_{*} E(0)}^{0, *}\left(E(0)_{*}, E(0)_{*} B P\right)=p^{-1} B P_{*},
$$

this gives rise to an exact sequence

$$
\begin{aligned}
0 \rightarrow \operatorname{Ext}_{B P_{*} B P}^{0, *}\left(B P_{*}, B P_{*} B P\right) & \longrightarrow p^{-1} B P_{*} \\
& \stackrel{\mathrm{d}_{1}}{\longrightarrow} \operatorname{Ext}_{E(1)_{*} E(1)}^{0, *}\left(E(1)_{*}, E(1)_{*} B P /\left(p^{\infty}\right)\right) \stackrel{\mathrm{d}_{1}}{\longrightarrow} \cdots
\end{aligned}
$$

From $\operatorname{CSS}(B P ; E(1))$ we obtain an exact sequence

$$
0 \rightarrow B P_{*} \longrightarrow p^{-1} B P_{*} \stackrel{\mathrm{d}_{1}}{\longrightarrow} \operatorname{Ext}_{E(1)_{*} E(1)}^{0, *}\left(E(1)_{*}, E(1)_{*} B P /\left(p^{\infty}\right)\right)
$$

implying that the Hurewicz homomorphism $B P_{*} /\left(p^{\infty}\right) \longrightarrow E(1)_{*} B P /\left(p^{\infty}\right)$ is a monomorphism. This in turn shows that the Hurewicz homomorphism $B P_{*} \longrightarrow E(1)_{*} B P$ is indivisible, hence is a split monomorphism by Lemma 3.2.

For $n \geqslant 1, \operatorname{CSS}(B P ; E(n))$ gives rise to an exact sequence

$$
\begin{aligned}
0 \rightarrow B P_{*} \longrightarrow p^{-1} B P_{*} \stackrel{\mathrm{d}_{1}}{\longrightarrow} \operatorname{Ext}_{E(1)_{*} E(1)}^{0, *}\left(E(1)_{*}, E(1)_{*} B P /\left(p^{\infty}\right)\right) \stackrel{\mathrm{d}_{1}}{\longrightarrow} \cdots \\
\ldots \stackrel{\mathrm{d}_{1}}{\longrightarrow} \operatorname{Ext}_{E(n)_{*} E(n)}^{0, *}\left(E(n)_{*}, E(n)_{*} B P /\left(p^{\infty}, v_{1}^{\infty}, \ldots, v_{n-1}^{\infty}\right)\right) .
\end{aligned}
$$


From this we obtain

$$
\mathrm{E}_{\infty}^{0,0, *} \cong \operatorname{Ext}_{E(n)_{*} E(n)}^{0, *}\left(E(n)_{*}, E(n)_{*} B P\right)=B P_{*},
$$

and together with Lemma 3.2, this proves the theorem.

Theorem 3.3. For $n \geqslant 1$,

$$
\operatorname{Ext}_{E(n)_{*} E(n)}^{s, *}\left(E(n)_{*}, E(n)_{*} B P\right) \cong \begin{cases}B P_{*} & \text { if } s=0, \\ B P_{*} /\left(p^{\infty}, v_{1}^{\infty}, \ldots, v_{n}^{\infty}\right) & \text { if } s=n, \\ 0 & \text { if } s \neq 0, n,\end{cases}
$$

Proof. This is deduced using the exact sequence derived from $\operatorname{CSS}(B P ; E(n))$. It only remains to identify Ext ${ }^{n}$ which we do by induction on $n$.

When $n=0, \operatorname{CSS}(B P ; E(0))$ has trivial $\mathrm{E}_{1}$-term

$$
\mathrm{E}_{1}^{0, t, *}(B P ; E(0))= \begin{cases}p^{-1} B P_{*} & \text { if } t=0 \\ 0 & \text { if } t \neq 0\end{cases}
$$

This gives rise to the exact sequence

$$
0 \rightarrow B P_{*} \longrightarrow p^{-1} B P_{*} \longrightarrow B P_{*} /\left(p^{\infty}\right) \rightarrow 0,
$$

where $B P_{*} /\left(p^{\infty}\right)$ is identified with the image of the differential $\mathrm{d}_{1}$ in $\operatorname{CSS}(B P ; E(1))$.

Consider the case $n=1$ and $\operatorname{CSS}(B P ; E(1))$. Here the $\mathrm{E}_{1}$-term is

$$
0 \rightarrow p^{-1} B P_{*} \stackrel{\mathrm{d}_{1}}{\longrightarrow} v_{1}^{-1} B P_{*} /\left(p^{\infty}\right) \rightarrow 0
$$

in which $d_{1}$ is the evident composite

$$
p^{-1} B P_{*} \longrightarrow B P_{*} /\left(p^{\infty}\right) \longrightarrow v_{1}^{-1} B P_{*} /\left(p^{\infty}\right) .
$$

Hence the $\mathrm{E}_{1}$-term fits into an exact sequence

$$
0 \rightarrow B P_{*} \longrightarrow p^{-1} B P_{*} \stackrel{\mathrm{d}_{1}}{\longrightarrow} v_{1}^{-1} B P_{*} /\left(p^{\infty}\right) \longrightarrow B P_{*} /\left(p^{\infty}, v_{1}^{\infty}\right) \rightarrow 0
$$

and the $\mathrm{E}_{\infty}$-term is

$$
\mathrm{E}_{\infty}^{1, r, *}(B P ; E(1))= \begin{cases}B P_{*} & \text { if } r=0 \\ B P_{*} /\left(p^{\infty}, v_{1}^{\infty}\right) & \text { if } r=1 \\ 0 & \text { if } r \neq 0,1\end{cases}
$$

If the stated result is correct for $n<m$, then for $n=m$ the $\mathrm{E}_{1}$-term is the complex

$$
\begin{aligned}
0 \rightarrow p^{-1} B P_{*} \stackrel{\mathrm{d}_{1}}{\longrightarrow} v_{1}^{-1} B P_{*} /\left(p^{\infty}\right) \stackrel{\mathrm{d}_{1}}{\longrightarrow} \cdots \\
\quad \ldots \stackrel{\mathrm{d}_{1}}{\longrightarrow} v_{n-1}^{-1} B P_{*} /\left(p^{\infty}, \cdots, v_{n-2}^{\infty}\right) \stackrel{\mathrm{d}_{1}}{\longrightarrow} v_{n}^{-1} B P_{*} /\left(p^{\infty}, \ldots, v_{n-1}^{\infty}\right) \rightarrow 0,
\end{aligned}
$$

in which the differentials are evident composites of the form

$$
v_{k}^{-1} B P_{*} /\left(p^{\infty} \ldots, v_{k-1}^{\infty}\right) \longrightarrow B P_{*} /\left(p^{\infty}, \ldots, v_{k-1}^{\infty}, v_{k}^{\infty}\right) \longrightarrow v_{k+1}^{-1} B P_{*} /\left(p^{\infty}, \ldots, v_{k}^{\infty}\right) .
$$

From this it is clear that

$$
\mathrm{E}_{\infty}^{n, t, *}(B P ; E(n))= \begin{cases}B P_{*} & \text { if } t=0 \\ B P_{*} /\left(p^{\infty}, v_{1}^{\infty}, \ldots, v_{n}^{\infty}\right) & \text { if } t=n \\ 0 & \text { if } t \neq 0, n\end{cases}
$$

This establishes the inductive step. 
It is interesting to note that the case $n=1$ gives the diagram of exact sequences

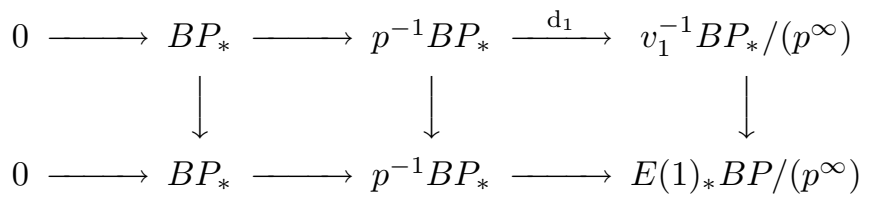

This shows that the composite map

$$
B P_{*} /\left(p^{\infty}\right) \longrightarrow v_{1}^{-1} B P_{*} /\left(p^{\infty}\right) \longrightarrow E(1)_{*} B P /\left(p^{\infty}\right)
$$

is a monomorphism. As this map agrees with the E(1)-theory Hurewicz map

$$
B P_{*} /\left(p^{\infty}\right) \longrightarrow E(1)_{*} B P /\left(p^{\infty}\right),
$$

the Hurewicz map $B P_{*} \longrightarrow E(1)_{*} B P$ is indivisible, hence is a split monomorphism. This is a reformulation of the classical Hattori-Stong Theorem.

One analogue of Hattori-Stong for $E(n)_{*} B P$ is that the $E(n)$-theory Hurewicz map $B P_{*} \longrightarrow$ $E(n)_{*} B P$ is indivisible; this follows from knowledge of the spectral sequence $\operatorname{CSS}(B P ; E(n))$. A more delicate consequence is that the homomorphism

$$
B P_{*} /\left(p^{\infty}, \ldots, v_{n-1}^{\infty}\right) \longrightarrow v_{n}^{-1} B P_{*} /\left(p^{\infty}, \ldots, v_{n-1}^{\infty}\right) \longrightarrow E(n)_{*} B P /\left(p^{\infty}, \ldots, v_{n-1}^{\infty}\right)
$$

is a monomorphism, hence

$$
B P_{*} /\left(p^{\infty}, \ldots, v_{n-2}^{\infty}\right) \longrightarrow E(n)_{*} B P /\left(p^{\infty}, \ldots, v_{n-2}^{\infty}\right)
$$

is $v_{n-1}$-indivisible.

\section{A change of Hopf algebroid spectral sequence}

We will make use of a spectral sequence concerning a morphism $\varphi:(A, \Gamma) \longrightarrow(B, \Sigma)$ of Hopf algebroids over a commutative unital ring $\mathbb{k}$.

Let $M$ be a left $\Gamma$-comodule and $N$ a left $\Sigma$-comodule. We give $\Gamma \otimes_{A} B$ and $M \otimes_{A} B$ their natural right $\Sigma$-comodule structures. We also have the cotensor product analogue of the adjunction formula of [5]. We say that a $A$ - $B$-bimodule $L$ is a $\Gamma$ - $\Sigma$-bicomodule if it admits left $\Gamma$-comodule and right $\Sigma$-comodule structures

$$
\Gamma \underset{A}{\otimes} L \stackrel{\psi_{L}}{\longleftarrow} L \stackrel{\psi_{R}}{\longrightarrow} L \underset{B}{\otimes} \Sigma
$$

which satisfy the compatibility condition

$$
\left(1 \otimes \psi_{R}\right) \psi_{L}=\left(\psi_{L} \otimes 1\right) \psi_{R}
$$

If $L$ is a $\Gamma-\Sigma$ bicomodule, then by coassociativity

$$
M \underset{\Gamma}{\square}(L \underset{\Sigma}{\square} N) \cong(M \underset{\Gamma}{\square} L) \underset{\Sigma}{\square} N,
$$

implying

$$
M \underset{\Gamma}{\square}((\Gamma \underset{A}{\otimes} B) \underset{\Sigma}{\square} N) \cong(M \underset{\Gamma}{\square}(\Gamma \underset{A}{\otimes} B)) \underset{\Sigma}{\square} N \cong(M \underset{A}{\otimes} B) \underset{\Sigma}{\square} N
$$

Theorem 4.1. If $M$ is a flat A-module, there is a spectral sequence

$$
\mathrm{E}_{2}^{s, t, *}(M, N)=\operatorname{Cotor}_{\Gamma}^{s, *}\left(M, \operatorname{Cotor}_{\Sigma}^{t, *}(\Gamma \underset{A}{\otimes} B, N)\right) \Longrightarrow \operatorname{Cotor}_{\Sigma}^{s+t, *}(M \underset{A}{\otimes} B, N)
$$

with differentials $\mathrm{d}_{r}: \mathrm{E}_{2}^{s, t, *}(M, N) \longrightarrow \mathrm{E}_{2}^{s+r, t-r+1, *}(M, N)$. 
Proof. This spectral sequence is constructed by Ravenel [6, theorem A1.3.11]. However, we feel it is illuminating to view it as a Grothendieck composite functor spectral sequence as discussed in $[\mathbf{7}, \mathbf{8}]$. For completeness we outline this description.

It is a standing assumption that for a Hopf algebroid $(A, \Gamma), \Gamma$ is flat as a left or right $A$-module. Given a $\mathbb{k}$-algebra homomorphism $A \longrightarrow B, \Gamma \otimes_{A} B$ is flat as a right $B$-module and $B \otimes_{A} \Gamma$ is flat as a left $B$-module. Also, if $M$ is a flat $A$-module the functors $\operatorname{Cotor}_{\Gamma}^{s}(M$, $(s \geqslant 0)$ is computable using resolutions of the second variable by weakly injective extended comodules.

Consider the following functors and their right derived functors:

$$
G: N \rightsquigarrow(\Gamma \underset{A}{\otimes} B) \underset{\Sigma}{\square} N, \quad \mathrm{R}^{t} G(\quad)=\operatorname{Cotor}_{\Sigma}^{t, *}\left(\Gamma{\underset{A}{\otimes}}^{\otimes} B, \quad\right),
$$

from the category of left $\Sigma$-comodules to left $\Gamma$-comodules, and

$$
F: L \rightsquigarrow M \underset{\Gamma}{\square} L, \quad \mathrm{R}^{s} F(\quad)=\operatorname{Cotor}_{\Gamma}^{s, *}(M, \quad),
$$

from the category of left $\Gamma$-comodules to $\mathbb{k}$-modules. Then by Equation (4.1),

$$
F G() \cong(M \underset{A}{\otimes} B) \underset{\Sigma}{\square}(\quad)
$$

with right derived functors

$$
\mathrm{R}^{n} F G(\quad)=\operatorname{Cotor}_{\Sigma}^{n, *}(M \underset{A}{\otimes} B, \quad) .
$$

For an extended $\Sigma$-comodule $\Sigma \otimes_{B} N_{0}$,

$$
G\left(\Sigma \underset{B}{\otimes} N_{0}\right)=(\Gamma \underset{A}{\otimes} B) \underset{\Sigma}{\square}\left(\Sigma \underset{B}{\otimes} N_{0}\right) \cong(\Gamma \underset{A}{\otimes} B) \underset{B}{\otimes} N_{0},
$$

which is an extended $\Gamma$-comodule. So for $s>0$,

$$
\mathrm{R}^{s} F\left(G\left(\sum \underset{B}{\otimes} N_{0}\right)\right)=0 .
$$

This is the condition required to obtain the Grothendieck Spectral Sequence

$$
\mathrm{E}_{2}^{s, t}(M, N)=\mathrm{R}^{s} F \circ \mathrm{R}^{t} G(N) \Longrightarrow \mathrm{R}^{s+t} F G(N) .
$$

In the following corollary, $\operatorname{Ext}_{\Gamma}$ is taken on the category of left $\Gamma$-comodules, while Ext $_{\Sigma}$ is taken on the category of right $\Sigma$-comodules.

Corollary 4.2. There is a spectral sequence

$$
\mathrm{E}_{2}^{s, t, *}=\operatorname{Ext}_{\Gamma}^{s, *}\left(A, \operatorname{Ext}_{\Sigma}^{t, *}(B, \Gamma \underset{A}{\otimes} B)\right) \Longrightarrow \operatorname{Ext}_{\Sigma}^{s+t, *}(B, B)
$$

with differentials $\mathrm{d}_{r}: \mathrm{E}_{2}^{s, t, *} \longrightarrow \mathrm{E}_{2}^{s+r, t-r+1, *}$.

Proof. Recalling the standard equivalences of functors

$$
\operatorname{Ext}_{\Gamma}^{s, *}(A,) \cong \operatorname{Cotor}_{\Gamma}^{s, *}(A,), \quad \operatorname{Ext}_{\Sigma}^{s, *}(B,) \cong \operatorname{Cotor}_{\Sigma}^{s, *}(, B),
$$

this follows from the Theorem with $M=A$ (with its right coaction twisted into a left action) and $N=B$.

As a particular case of this result, if $\Gamma \otimes_{A} B$ is a relatively injective right $\Sigma$-comodule then

$$
\operatorname{Ext}_{\Sigma}^{n, *}(B, B) \cong \operatorname{Ext}^{n, *}\left(A,\left(\Gamma \otimes_{A}^{\otimes} B\right) \square_{\Sigma} B\right) \cong \operatorname{Ext}^{n, *}\left(A,(\Gamma \underset{A}{\otimes} B) \square_{\Sigma} B\right),
$$

which is the Shapiro Lemma of Miller \& Ravenel [5].

Clarke [3] pointed out that the morphism of Hopf algebroids

$$
\tau:\left(M U_{*}, M U_{*} M U\right) \longrightarrow\left(K U_{*}, K U_{*} K U\right)
$$


induced by the Todd orientation $M U \longrightarrow K U$ gives an example for which this assumption fails since $\operatorname{Ext}_{K U_{*} K U}^{1, *}\left(K U_{*}, K U_{*}\right)$ has infinitely many elements surviving from

$$
\operatorname{Ext}_{M U_{*} M U}^{0, *}\left(M U_{*}, \operatorname{Ext}_{K U_{*} K U}^{1, *}\left(K U_{*}, M U_{*} K U\right)\right)
$$

in the relevant spectral sequence. At each prime $p$ this can also be seen by considering the morphism

$$
\tau_{1}:\left(B P_{*}, B P_{*} B P\right) \longrightarrow\left(E(1)_{*}, E(1)_{*} E(1)\right) .
$$

induced by the natural orientation $B P \longrightarrow E(1)$. More generally, we will describe the relevant spectral sequence for each of the natural maps

$$
\tau_{n}:\left(B P_{*}, B P_{*} B P\right) \longrightarrow\left(E(n)_{*}, E(n)_{*} E(n)\right) .
$$

Let $\operatorname{SS}(n)$ denote the spectral sequence of Corollary 4.2 associated with $\tau_{n}$.

Theorem 4.3. For $n \geqslant 1, \operatorname{SS}(n)$ has

$$
\mathrm{E}_{2}^{s, t, *}= \begin{cases}\operatorname{Ext}_{B P_{*} B P}^{s, *}\left(B P_{*}, B P_{*}\right) & \text { if } t=0, \\ \operatorname{Ext}_{B P_{*} B P}^{s, *}\left(B P_{*}, B P_{*} /\left(p^{\infty}, v_{1}^{\infty}, \ldots, v_{n}^{\infty}\right)\right) & \text { if } t=n, \\ 0 & \text { if } t \neq 0, n .\end{cases}
$$

For $k>0$ and $p \nmid k$,

$$
\mathrm{E}_{2}^{0,1,-2 k(p-1) p^{r}}=\operatorname{Ext}_{B P_{*} B P}^{0,-2 k(p-1) p^{r}}\left(B P_{*}, B P_{*} /\left(p^{\infty}, v_{1}^{\infty}\right)\right)
$$

contains a summand isomorphic to $\mathbb{Z} / p^{r+1}$, having as generator the class represented in the usual chromatic notation by $\frac{1}{p^{r+1} v_{1}^{k p^{r}}}$. Such classes are non-vanishing infinite cycles which generate the cyclic groups in $\mathrm{E}_{\infty}$ observed by Clarke. There are of course other elements in this group, for example $\frac{v_{2}}{p v_{1}}$. Such elements are killed by the differential $\mathrm{d}_{2}$ and in fact

$$
\mathrm{d}_{2}: \operatorname{Ext}_{B P_{*} B P}^{s, *}\left(B P_{*}, B P_{*} /\left(p^{\infty}, v_{1}^{\infty}\right)\right) \longrightarrow \operatorname{Ext}_{B P_{*} B P}^{s+2, *}\left(B P_{*}, B P_{*} /\left(p^{\infty}, v_{1}^{\infty}\right)\right)
$$

is the composition of chromatic coboundaries

$$
\begin{aligned}
\operatorname{Ext}_{B P_{*} B P}^{s, *}\left(B P_{*}, B P_{*} /\left(p^{\infty}, v_{1}^{\infty}\right)\right) \stackrel{\delta_{1}}{\longrightarrow} \operatorname{Ext}_{B P_{*} B P}^{s+1, *}\left(B P_{*}, B P_{*} /\left(p^{\infty}\right)\right) \\
\stackrel{\delta_{0}}{\longrightarrow} \operatorname{Ext}_{B P_{*} B P}^{s+2, *}\left(B P_{*}, B P_{*}\right) .
\end{aligned}
$$

Thus we have

$$
\mathrm{d}_{2}\left(\frac{v_{2}}{p v_{1}}\right)=\beta_{1}
$$

Similarly, when $n \geqslant 1$, the differential $\mathrm{d}_{n+1}$ is a composite of chromatic coboundaries

$$
\begin{aligned}
\operatorname{Ext}_{B P_{*} B P}^{s, *}\left(B P_{*}, B P_{*} /\left(p^{\infty}, v_{1}^{\infty}, \ldots, v_{n}^{\infty}\right)\right) \stackrel{\delta_{n}}{\longrightarrow} & \\
\operatorname{Ext}_{B P_{*} B P}^{s+1, *}\left(B P_{*}, B P_{*} /\left(p^{\infty}, v_{1}^{\infty}, \ldots, v_{n-1}^{\infty}\right)\right) & \stackrel{\delta_{n-1}}{\longrightarrow} \cdots \\
\cdots & \stackrel{\delta_{0}}{\longrightarrow} \operatorname{Ext}_{B P_{*} B P}^{s+n+1, *}\left(B P_{*}, B P_{*}\right) .
\end{aligned}
$$

The elements

$$
\frac{1}{p^{r_{0}} v_{1}^{r_{1}} \ldots v_{n}^{r_{n}}} \in \operatorname{Ext}_{B P_{*} B P}^{0, *}\left(B P_{*}, B P_{*} /\left(p^{\infty}, v_{1}^{\infty}, \ldots, v_{n}^{\infty}\right)\right) \quad\left(r_{j} \geqslant 1\right),
$$

are non-vanishing infinite cycles, corresponding to non-trivial elements of

$$
\operatorname{Ext}_{E(n)_{*} E(n)}^{n, *}\left(E(n)_{*}, E(n)_{*}\right) .
$$


The general picture is that there is an exact sequence of the form

$$
\begin{aligned}
0 \rightarrow F^{0, s, *} \longrightarrow \operatorname{Ext}_{B P_{*} B P}^{s, *}\left(B P_{*}, B P_{*} /\left(p^{\infty}, v_{1}^{\infty}, \ldots, v_{n}^{\infty}\right)\right) \stackrel{\delta_{0} \cdots \delta_{n}}{\longrightarrow} \\
\operatorname{Ext}_{B P_{*} B P}^{s+n+1, *}\left(B P_{*}, B P_{*}\right) \longrightarrow F^{n, s+n+1, *} \rightarrow 0,
\end{aligned}
$$

where the filtration groups $F^{k, *, *}$ fit into short exact sequences

$$
0 \rightarrow F^{0, s, *} \longrightarrow F^{n, s, *} \longrightarrow \operatorname{Ext}_{E(n)_{*} E(n)}^{s, *}\left(E(n)_{*}, E(n)_{*}\right) \rightarrow 0 .
$$

\section{References}

[1] A. Baker, A version of the Landweber filtration theorem for $v_{n}$-periodic Hopf algebroids, Osaka J. Math. 32 (1995), 689-99.

[2] A. Baker, On the Adams $\mathrm{E}_{2}$-term for elliptic cohomology, to appear in Proceedings of the 1999 Boulder Conference in Contemp. Math.

[3] F. Clarke, On $\operatorname{Ext}_{K_{*}(K)}\left(\pi_{*}(K), K_{*}(M U)\right)$, Proc. Amer. Math. Soc. 74 (1979), 156-160.

[4] M. Hovey \& H. Sadofsky, Invertible spectra in the $E(n)$-local stable homotopy category, J. London Math. Soc. 60 (1999), 284-302.

[5] H. R. Miller \& D. C. Ravenel, Morava stabilizer algebras and localization of Novikov's $\mathrm{E}_{2}$-term, Duke Math. J. 44 (1977), 433-47.

[6] D. C. Ravenel, Complex Cobordism and the Stable Homotopy Groups of Spheres, Academic Press (1986).

[7] S. Shatz, Profinite Groups, Arithmetic and Geometry, Annals of Mathematics Studies 67 (1972), Princeton University Press.

[8] C. A. Weibel, An Introduction to Homological Algebra, Cambridge University Press (1994).

This article may be accessed via WWW at http://www.rmi.acnet.ge/hha/ or by anonymous ftp at ftp://ftp.rmi.acnet.ge/pub/hha/volumes/2000/n3/n3.(dvi,ps,dvi.gz,ps.gz)

Andrew Baker a.baker@maths.gla.ac.uk http://www.maths.gla.ac.uk/ ajb

Department of Mathematics

University of Glasgow

Glasgow G12 8QW

Scotland. 